\title{
Sterile Neutrino as a Bulk Neutrino
}

\author{
R. N. Mohapatra ${ }^{1 *}$, and A. Pérez-Lorenzana ${ }^{1,2 \dagger}$ \\ ${ }^{1}$ Department of Physics, University of Maryland, College Park, MD, 20742, USA \\ ${ }^{2}$ Departamento de Física, Centro de Investigación y de Estudios Avanzados del I.P.N. \\ Apdo. Post. 14-740, 07000, México, D.F., México.
}

(October, 1999)

\begin{abstract}
If light sterile neutrinos are needed to understand the neutrino puzzles, as is currently indicated, a major theoretical challenge is to understand why its mass is so small. It is a more serious problem than understanding the small mass of the familiar neutrinos. We discuss a new way to solve this problem by identifying the sterile neutrino as gauge neutral fermion propagating in the bulk of a higher dimensional theory, with bulk size of order of a millimeter. The smallness of its mass is then a consequence of the size of the extra dimension and does not need the introduction of new symmetries. We present a realistic model for neutrino masses and mixings that implements this idea.
\end{abstract}

\section{INTRODUCTION}

Recent experimental data on atmospheric neutrinos from the Super-Kamiokande [1] collaboration has provided conclusive evidence for neutrino oscillations and confirms earlier indications of such oscillations from other experiments [2]. In addition there is also evidence for neutrino oscillations from the solar neutrino deficit observed by Kamiokande, Homestake, Gallex, Sage and Super-Kamiokande [3, [4], and direct observation of $\nu_{\mu} \rightarrow \nu_{e}\left(\bar{\nu}_{\mu} \rightarrow \bar{\nu}_{e}\right)$ in the LSND [5] experiment. All these suggest nonvanishing masses for at least two of the three standard neutrinos and opens the window to explore physics beyond the standard model.

It has been realized that to explain all three evidences for neutrino oscillations three different mass differences are needed, while only two can be obtained with three neutrinos. Solar neutrino data requires [6]

$$
\begin{aligned}
\Delta m_{\text {sol }}^{2} & \sim 3 \times 10^{-6}-1.2 \times 10^{-5} e V^{2}, \\
\sin ^{2} 2 \theta_{\text {sol }} & \sim 3 \times 10^{-3}-1.5 \times 10^{-2}
\end{aligned}
$$

\footnotetext{
*e-mail:rmohapat@physics.umd.edu

†e-mail:aplorenz@Glue.umd.edu
} 
for the small mixing angle MSW [7] solution. Atmospheric neutrinos seems to prefer $\nu_{\mu}-\nu_{\tau}$ oscillations with a maximal mixing [8]:

$$
\begin{aligned}
\Delta m_{a t m}^{2} & \sim 4 \times 10^{-4}-5 \times 10^{-3} e V^{2}, \\
\sin ^{2} 2 \theta_{a t m} & \sim .76-1 .
\end{aligned}
$$

Finally, the LSND results along with other constraints from KARMEN [9], Bugey [10] and E776 at BNL [11] suggest

$$
\begin{aligned}
\Delta m_{e \mu}^{2} & \sim .2-2 e V^{2}, \\
\sin ^{2} 2 \theta_{e \mu} & \sim 2 \times 10^{-3}-4 \times 10^{-2} .
\end{aligned}
$$

A simple explanation of these parameters could be obtained if there is a sterile neutrino [12 (which is a light neutrino that does not couple to the Standard Model particles). The existence of a light sterile neutrino however poses a major theoretical challenge. It is more problematic to understand its small mass than to understand the small mass of the familiar neutrinos. This is because, the familiar neutrinos are standard model gauge nonsinglets; therefore their mass can at most be of order of the weak scale. On the other hand the sterile neutrinos being standard model singlets, their apriori mass could be of order of the Planck scale- some sixteen orders of magnitude higher.

Several scenarios involving sterile neutrino have been studied in the literature [13 16] where the general strategy has been to invoke new symmetries of different kinds to explain its small mass. It is clearly too early to say which if any of these approaches is the correct one. Here we explore a completely different approach to its mass. We consider the possibility that the sterile neutrino is a massless Dirac bulk fermion in a higher dimensional brane-bulk picture with at least one large extra dimension. The bulk fermion has Kaluza-Klein (KK) excitations whose spacing is given by the inverse size of the bulk. If the bulk size is large (say a millimeter) as has been speculated recently [17], then the sterile neutrino (i.e. the bulk neutrino) mass is automatically small without the need for any extra symmetries. In recent days, interest in such theories (i.e. theories with large extra dimensions) has been heightened from independent experimental considerations since they lead to deviations from Newton's inverse square law of gravity at small distances. In fact the maximum bulk size believed to be at the boundary of gravity experiments is a millimeter, which corresponds to sterile neutrino masses of milli-eV's. This value of masses is precisely what is interesting for solving the solar neutrino problem providing an intriguing connection between the neutrino experiments and those searching for deviations from Newton's law in the millimeter range.

In the brane-bulk models with large extra dimensions, the smallness of the KK excitations of the bulk fermion mass is related to the size of the extra dimensions as just explained. On the other hand the small mass of familiar neutrinos arises from the following fact. There is a suppression of the coupling between neutrinos living on the bulk with those fixed on our four dimensional world [18 21] which arises naturally from normalization of the bulk field. As far as the oscillations between the brane and bulk neutrinos is concerned, it is dominated by the mass of the lowest KK mode of the bulk neutrino. Thus one has the right parameter range to give an explanation for the solar neutrino deficit.

Two distinctive scenarios which involve bulk neutrinos have been outlined in recent literature. The first uses only the standard model left handed neutrinos in the brane and 
one or more bulk fermions [18,19] and a second one that has both the left and right handed neutrinos in the brane in combination with a bulk neutrino [21]. In the second case, one could invoke the seesaw mechanism [22] in the brane to understand the smallness of the neutrino masses; therefore the higher dimensional physics need not play a role. Of course it could happen that implementation of seesaw mechanism is impossible due to group theory of the model [21] in which case the extra dimension as well as the string scale will play a role in neutrino physics.

It must however be emphasized that even though mechanisms to understand small neutrino masses have been outlined, no attempt has been made to construct realistic models that explain neutrino observations using the property of higher dimensional physics. It is the goal of this paper to make an attempt in this direction and in particular construct a model involving a sterile neutrino that explains the present neutrino data.

We first study the models without right handed neutrinos and point out that even though the mass of the sterile neutrino is naturally small in these models, several unnatural assumptions are needed if we are to obtain a desirable mixing and mass pattern for understanding observations. We then study models with both left and right handed neutrinos in the brane with seesaw mechanism that explains the small masses for two of the known neutrinos and include a bulk neutrino with one extra dimension being in the submillimeter range to play the role of the sterile neutrino. Since the masses of many of the low KK excitations are then in the range of interest for solar neutrino oscillations, the key question is whether the mixings pattern is such as to be of interest in solving the neutrino puzzles without at the same time contradicting known observations. In particular, we explore whether only the lowest mode of this bulk neutrino can play the role of the sterile neutrino. The advantage of this approach over other models for the sterile neutrino is that smallness of its mass is now a geometrical rather than a symmetry effect. The nontriviality of the problem arises from the fact that the bulk neutrino of course has infinite number of excitations and one has to tackle the mathematical problem of extracting the physical masses and mixings of neutrinos from this complex situation and study whether one has a solution to the neutrino puzzles.

Our basic results in the second case are the following: the lightest eigenstate which is predominantly the electron neutrino is massless and it mixes with the first excited KK mode of the bulk neutrino, which has mass of order $R^{-1}$. We use this to solve the solar neutrino deficit via the small angle MSW mechanism. This requires the size of the extra dimension to be in the micro to millimeter range and a string scale which is in the range of $10^{8} \mathrm{GeV}$. Only one large extra dimension is sufficient in our discussion. The smallness of the $\nu_{\mu, \tau}$ is due to seesaw mechanism in the brane that uses intermediate seesaw scales (of the order of the string scale or so). The maximal $\nu_{\mu}-\nu_{\tau}$ mixing needed for atmospheric oscillations owes its origin to the texture of the right handed neutrino matrix. The LSND data is explained in a natural way via small mixing between generations. Thus the new contribution of this paper is the identification of the lowest KK mode of the bulk neutrino as a viable sterile neutrino candidate and its embedding into a realistic four neutrino gauge theory framework.

\section{MODELS WITHOUT RIGHT HANDED NEUTRINOS}

Let us start by summarizing the simplest mechanism to produce neutrino masses using extra dimensions as discussed in Ref. [19]. One assumes that all the Standard Model particles 
are localized on a brane embedded in the bulk of larger dimensions. The conservation of gauge flux then implies that, besides gravity, the only fields that could propagate in the extra dimensions are standard model gauge singlets. Their coupling with the brane fields is naturally suppressed by the volume factor $\frac{M_{*}}{M_{P \ell}}$, where, from the observed strength of gravity the Plank scale and the string scale $M_{*}$ are related by

$$
M_{P \ell}^{2}=M_{*}{ }^{n+2} V_{n}
$$

with $V_{n}$ the volume of the extra space. We will assume that one of the radii of the compact extra dimensions, $\mathrm{R}$, is larger. Now, by including the coupling of a bulk neutrino $\nu_{B}\left(x^{\mu}, y\right)$ to the standard model lepton doublet $L\left(x^{\mu}, y=0\right)$ we get the following terms which are responsible for the neutrino mass in this model

$$
\mathcal{S}=\kappa \int d^{4} x \bar{L} H \nu_{B R}(x, y=0)+\int d^{4} x d y \bar{\nu}_{B L}(x, y) \partial_{5} \nu_{B R}(x, y)+\text { h.c. }
$$

where $H$ is the Higgs doublet, $L$ is the lepton doublet and $\kappa$ is the suppressed Yukawa coupling

$$
\kappa=h \frac{M_{*}}{M_{P \ell}} .
$$

By introducing the Fourier expansion of the bulk field we may write down the Dirac mass terms in (4) as

$$
\left(\bar{\nu}_{e L} \bar{\nu}_{B L}^{\prime}\right)\left(\begin{array}{cc}
m & \sqrt{2} m \\
0 & \partial_{5}
\end{array}\right)\left(\begin{array}{c}
\nu_{0 B} \\
\nu_{B R}^{\prime}
\end{array}\right)
$$

where $\nu_{B}^{\prime}$ embodies in a compact way the KK excitations along the fifth dimension and $m=\kappa v$ is the mass term produced by the vacuum expectation value, $v$, of the Higgs field. The entry $\sqrt{2} m$ has to be interpreted as an (infinite) row vector of the form $\sqrt{2} m(1,1, \cdots)$. The operator $\partial_{5}$ represents the diagonal and infinite mass matrix of the KK modes. The eigenvalues of this operator are $n \mu_{0}$, with $\mu_{0}=1 / R$. For $m \ll \mu_{0}$ the mixing angle of the standard neutrino with the n-th bulk mode is [19]

$$
\tan \theta_{n} \approx \frac{\xi}{n} ; \quad \text { with } \quad \xi=\sqrt{2} m R
$$

Then, the standard neutrino has a mass $m$ and oscillations into bulk neutrino are present dominated by the first exited mode of mass $\mu_{0}$. An explanation to the solar neutrino problem then follows if one assumes MSW mechanism with $\mu_{0} \simeq 10^{-3} \mathrm{eV}(R \simeq 0.2 \mathrm{~mm})$. The question now is how one can extend the model to incorporate the atmospheric data and the LSND results.

First thing to note is that in this scenario, the mixing between neutrinos of different generations is expected to be produced through the couplings with the bulk neutrino only. However, even if the bulk neutrino is not blind and couples with a different Yukawa coupling to each brane neutrino, the mass matrix will produce two massless neutrinos which decouple from the oscillation pattern. Therefore, there is no room for understanding the atmospheric and LSND data in this framework. 
Let us now see if this problem can be cured by including three bulk neutrinos. The most general Dirac mass in this case may be written, after a proper rotation of the bulk fields, as

$$
\mathcal{L}=\bar{\nu}_{L} \cdot V M_{D} \cdot \nu_{B R}(y=0)+\int d y \bar{\nu}_{B L} \cdot \partial_{5} \cdot \nu_{B R}+\text { h.c. }
$$

with $\nu_{L}=\left(\nu_{e}, \nu_{\mu}, \nu_{\tau}\right)_{L} ; \nu_{B}=\left(\nu_{B}^{1}, \nu_{B}^{2}, \nu_{B}^{3}\right) ; M_{D}=\operatorname{Diag}\left(m_{1}, m_{2}, m_{3}\right)$; and where the unitary matrix $V$ depends on the texture of the Yukawa couplings to bulk neutrinos. The mass parameters in $M_{D}$ are small numbers of the order of $\mathrm{eV}$ or so and are given by the eigenvalues of the Yukawa matrix multiplied by $v$. Since the extra dimensional volume suppression is present, it is not unnatural to choose one of the masses to be in the range of $10^{-4} \mathrm{eV}$ as in (6) to solve the solar neutrino puzzle.

After rotating the standard sector by $V$, the last expression simplifies to the form

$$
\mathcal{L}=\sum_{a=1,2,3}\left[m_{a} \bar{\nu}_{L}^{a} \nu_{B R}^{a}(y=0)+\int d y \bar{\nu}_{B L}^{a} \partial_{5} \nu_{B R}^{a}+\text { h.c. }\right]
$$

Therefore, in this picture the pattern of neutrino oscillations follows the same as before with a generational mixing given by $V$, which must provide atmospheric and LSND mixings. However a proper understanding of the neutrino puzzles would need three independent mass parameters. At first glance we would be tempted to believe that these masses could be those involved in (10). However, this is not the case. As we shall show below the heaviest eigenstate $\nu_{L}^{a}$ has a mass $\tilde{m}_{a}=\min \left\{\mu_{0} / 2, m_{a}\right\}$. Thus, if solar neutrino data is assumed to be solved by $\nu_{e}-\nu_{B}$ oscillation, this would require that $\mu_{1} \simeq 10^{-3} \mathrm{eV}$. As a result other mass differences in this picture become too small to solve the atmospheric and LSND data. If on the other hand, we assumed that it is the atmospheric neutrino puzzle which is solved by using the bulk neutrino, then we would choose $\mu_{1} \simeq 0.06 \mathrm{eV}$; one could envision solving the solar neutrino puzzle by $\nu_{e}-\nu_{\mu}$ oscillation, with the relevant mass difference coming from small Yukawa couplings. Then we would be unable to explain the LSND data.

Thus it appears that the only way out is to assume that the bulk neutrinos come from different branes with different sizes: $\mu_{0} \simeq 10^{-3}$, and $\mu_{2,3} \simeq 1 \mathrm{eV}$, with $\Delta m_{\text {atm }}^{2}=\mu_{2}-\mu_{3}$. The challenge in such a scenario is to explain how the mass terms can be diagonalized simultaneously, a fact used to obtain Eq. (10).

To prove our previous statement, let us consider again the mass matrix (7). In this compact notation it is simple to get the exact solution for the eigensystem even when the mass matrix is infinite. First the characteristic equation is given by

$$
\left(m_{n}^{2}-\partial_{5}^{2}\right)\left[m_{n}^{2}-m^{2}+\frac{2 m_{n}^{2} m^{2}}{\partial_{5}^{2}-m_{n}^{2}}\right]=0,
$$

where $m_{n}$ is the mass eigenvalue and a sum on the last term is implicit. This expression translates into the same result that was obtained before in references [18,19]

$$
m_{n}=\pi m^{2} R \cot \left(\pi m_{n} R\right) .
$$

The eigenstates are thus given symbolically by

$$
\tilde{\nu}_{n L}=\frac{1}{N_{n}}\left[\nu_{L}+\frac{\sqrt{2} m \partial_{5}}{m_{n}^{2}-\partial_{5}^{2}} \nu_{B L}^{\prime}\right],
$$


where the normalization factor $N_{n}$ is easily computed to be

$$
N_{n}^{2}=1+2 m^{2} R^{2} \sum_{k=1}^{\infty} \frac{k^{2}}{\left(k^{2}-m_{n}^{2} R^{2}\right)^{2}}=\frac{1}{2}\left[1+(\pi m R)^{2}+\left(\frac{m_{n}}{m}\right)^{2}\right] .
$$

Now we may express $\nu_{L}$ in terms of the massive modes just as

$$
\nu_{L}=\sum_{n=0}^{\infty} \frac{1}{N_{n}} \tilde{\nu}_{n L}
$$

Since $N_{n}$ has a minimum for the lowest value of $m_{n}$, the main component of $\nu_{L}$ is always the lightest mode $\tilde{\nu}_{0 L}$. Notice from (12) that when $m R \ll 1$, the eigenvalues comes out to be $m_{0}=m$ and $m_{n}=n \mu_{0}$ for $n>0$, and $N_{n} \approx m_{n} / \sqrt{2} m$, thus, we recover the mixing angle (8). However, in the other limit, when $m R \gg 1$ the lowest eigenvalues are shifted down to $(2 n+1) \mu_{0} / 2$. Then the main component of $\nu_{L}$ will have a mass of $\mu_{0} / 2$ and the mixing angle will be in general $\tan \theta_{n}=N_{0} / N_{n}$.

In consequence, irrespective of the hierarchy of the masses in $M_{D}$, a solution to the neutrino puzzles with bulk neutrinos needs localization of the three bulk neutrinos in different branes with extra assumptions about the Yukawa couplings.

\section{MODELS WITH RIGHT HANDED NEUTRINOS IN THE BRANE}

We now proceed to consider the second class of models where we include both the left and the right handed neutrinos in the same brane and a blind bulk neutrino which only couples with the right handed neutrinos due to either gauge symmetries 21] or asymmetric boundary conditions in the bulk [23].

The simplest gauge model where this scenario is realized is the left-right symmetric model where the right handed symmetry is broken by the doublet Higgs bosons $\chi_{R}(1,2,1)$, where the number inside the parenthesis correspond to the quantum numbers under $S U(2)_{L} \times$ $S U(2)_{R} \times U(1)_{B-L}$. The relevant terms of the action for one generation are

$$
\mathcal{S}=\int d^{4} x\left[\kappa \bar{L} \chi_{L} \nu_{B}(y=0)+\kappa \bar{R} \chi_{R} \nu_{B}(y=0)+h \bar{L} \phi R\right]+\int d^{4} x d y \bar{\nu}_{B} \Gamma^{5} \partial_{5} \nu_{B}+\text { h.c. }
$$

As discussed in reference [21], by setting $\left\langle\chi_{R}^{0}>=v_{R}\right.$ and $<\chi_{L}^{0}>=0$, the following Dirac neutrino mixing matrix is obtained

$$
\left(\begin{array}{lll}
\bar{\nu}_{e L} & \bar{\nu}_{0 B L} & \bar{\nu}_{B L}^{\prime}
\end{array}\right)\left(\begin{array}{cc}
h v & 0 \\
\kappa v_{R} & 0 \\
\sqrt{2} \kappa v_{R} & \partial_{5}
\end{array}\right)\left(\begin{array}{c}
\nu_{e R} \\
\nu_{B R}^{\prime}
\end{array}\right)
$$

with $\nu_{0 B L}$ being the zero mode and $\nu_{B}^{\prime}$ representing the exited modes as before. (Let us note parenthetically that in general a nonvanishing value for $\left\langle\chi_{L}^{0}\right\rangle$ could be expected from the potential, so we should assume either that this vev is small so that its contribution to neutrino masses is negligible or that the bulk neutrino breaks explicitly the parity symmetry so the coupling with $L$ is zero as advocated in Ref. [23].) Now, provided that $\kappa v_{R} \gg h v \simeq$ few $\mathrm{MeV}$, a massless field which is predominantly the electron neutrino appears. Since 
$\kappa \simeq \frac{M^{*}}{M_{P \ell}}$, this constraint implies that $M_{*}$ must be as large as $10^{8} \mathrm{GeV}$ or so. Oscillations into bulk neutrino will now result, again dominated by the lowest mass of the bulk modes [21], implying that the largest radius of the extra dimensions should still be at the millimeter range.

Let us now look at the complete picture for three generations. The more general mass terms in this class of models are

$$
\begin{aligned}
\mathcal{L}= & \bar{\nu}_{L} \cdot \mathcal{M}_{L L} \cdot \nu_{L}+\bar{\nu}_{L} \cdot M_{D} \cdot \nu_{R}+\bar{\nu}_{R} \cdot M_{N} \cdot \nu_{R} \\
& +\bar{\nu}_{R} \cdot \mathbf{m} \cdot \nu_{B L}(y=0)+\int d y \bar{\nu}_{B L} \cdot \partial_{5} \cdot \nu_{B R}+h . c .
\end{aligned}
$$

In last equation $\mathcal{M}_{L L}$ represent the Majorana mass terms of the left handed neutrinos. For simplicity we will assume those terms to be zero. $M_{N}$ represents the Majorana matrix for the right handed neutrinos which in the left-right models arises from the vev of a $B-L=2$ $S U(2)_{R}$ triplet and $\mathbf{m}^{\dagger}=m(1,1,1)$ are the universal couplings of the bulk neutrinos to the right handed neutrinos. The mass matrix now has the profile

$$
\left(\begin{array}{ccccc}
0 & M_{D} & 0 & 0 & 0 \\
M_{D}^{\dagger} & M_{N} & \mathbf{m} & \sqrt{2} \mathbf{m} & 0 \\
0 & \mathbf{m}^{\dagger} & 0 & 0 & 0 \\
0 & \sqrt{2} \mathbf{m}^{\dagger} & 0 & 0 & \partial_{5} \\
0 & 0 & 0 & \partial_{5} & 0
\end{array}\right)
$$

in the basis $\left(\nu_{L}, \nu_{R}, \nu_{0 B L}, \nu_{B L}^{\prime}, \nu_{B R}^{\prime}\right)$. Notice that this is really an infinite matrix with the entry $\sqrt{2} \mathbf{m}$ being the universal coupling of $\nu_{R}$ to the exited bulk modes $\nu_{n B L}$, then, this is an infinite matrix itself given by $\sqrt{2} \mathbf{m} \times(1,1, \cdots)$. We have used the two component notation and used subscripts $L, R$ as labels for corresponding states rather than helicity projection operators. Notice that $\nu_{0 B R}$ is massless and is therefore not included in the above mass matrix. As a result, this matrix has odd number of rows and columns and has one zero eigenvalue. The corresponding state is identified due to its flavor content to be the close to the $\nu_{e}$ state. The elements of $M_{N}$ are expected to be large, $\leq M_{*}$, while those in $M_{D}$ as well as $m$ are of the order of some $\mathrm{MeV}$. This via the usual seesaw mechanism leads to light eigenstates.

We can write down the general mixing matrix for the "four neutrino" states as:

$$
\left(\begin{array}{c}
\nu_{e} \\
\nu_{\mu} \\
\nu_{\tau} \\
\nu_{B}
\end{array}\right)_{L}=U \cdot\left(\begin{array}{l}
\nu_{1} \\
\nu_{2} \\
\nu_{3} \\
\nu_{4}
\end{array}\right)_{L}
$$

where the neutrinos in the right hand side are the mass eigenmodes, and $\nu_{4}$ represent the mass eigenstates of the KK tower. The active neutrinos will contain in general suppressed contributions from the complete KK tower, then, only the lowest mode is expected to contribute substancially and we will at the end identify this mode with $\nu_{4}$. We will show that in this picture, $\left|U_{e 1}\right| \approx 1$ and $\left|U_{e 4}\right|>\left|U_{e 2,3}\right| ; \nu_{1}$ being the lightest element of the spectrum with zero mass. If the masses of $\nu_{2,3}$ are in the $\mathrm{eV}$ range with a mass difference square of the the order of $\Delta m_{a t m}^{2}$, we can solve the atmospheric neutrino puzzle if $\left|U_{\mu 2}\right| /\left|U_{\mu 3}\right| \sim\left|U_{\tau 2}\right| /\left|U_{\tau 3}\right| \sim 1$, 
with the decoupling of the bulk modes from this sector; thus we would need $\left|U_{\mu, \tau 4}\right| \ll 1$ and $\left|U_{B 4}\right| \approx 1$. Finally to explain the LSND data, we need $\left|U_{\mu 1}\right| \sim\left|U_{e 2}\right| \gg\left|U_{e 3, \mu 4}\right|$. We will show below that all these features emerge only from the simple choice of the seesaw matrix $M_{N}$ as is commonly done in most model building [24].

Let us see how this scenario is realized in our picture. First note that the mass matrix in (19) has a zero mass left handed eigen mode, $\nu_{1 L}$, which is a linear combination of $\nu_{L}$ and $\nu_{0 B L}$ given by the solution to the matrix equation

$$
\left(M_{D}^{\dagger} \mathbf{m}\right) \cdot \mid \nu_{1 L}>=0
$$

where $\left(M_{D}^{\dagger} \mathbf{m}\right)$ is a $4 \times 3$ matrix. If we want this lightest eigenstate to be predominantly the electron neutrino to maintain the observed universality in charged current weak interactions, we must demand that in the expression for $\nu_{1 L}$,

$$
\nu_{1 L}=\frac{1}{N}\left[\nu_{e L}+\delta \nu_{\mu L}+\Delta \nu_{\tau L}-\epsilon \nu_{0 L}\right]
$$

we must have $\delta, \Delta, \epsilon \ll 1$. If we make the simplest choice for $M_{D}=\operatorname{Diag}\left(m_{1}, m_{2}, m_{3}\right)$, with the natural hierarchy $m_{1}<m_{2}<m_{3}$, we get $\delta=m_{1} / m_{2}>\Delta=m_{1} / m_{3}$ and $\epsilon=m_{1} / m$. We then may set $1>\epsilon>\delta \gg \Delta$ to get $\sin ^{2} 2 \theta_{\text {sol }} \simeq \epsilon^{2}$, and $\delta$ is going to produce the mixing for the LSND data.

After the extraction of the zero mass term (22) we may procceed to decouple the right handed fields which get large masses via $M_{N}$. The mass matrix for light fields then reduces to

$$
\left(\begin{array}{ccc}
M_{L} M_{N}^{-1} M_{L}^{\dagger} & \sqrt{2} M_{L} M_{N}^{-1} \mathbf{m} & 0 \\
\sqrt{2} \mathbf{m}^{\dagger} M_{N}^{-1} M_{L}^{\dagger} & 2 \mathbf{m}^{\dagger} M_{N}^{-1} \mathbf{m} & \partial_{5} \\
0 & \partial_{5} & 0
\end{array}\right),
$$

in the basis $\left(\nu_{0 B L}, \nu_{\mu L}, \nu_{\tau L}, \nu_{B L}^{\prime}, \nu_{B R}^{\prime}\right)$; with $M_{L}$ of the form

$$
M_{L}=\left(\begin{array}{ccc}
m & m & m \\
0 & m_{2} & 0 \\
0 & 0 & m_{3}
\end{array}\right)
$$

where we have neglected those contributions that are proportional to the small parameters $\epsilon, \delta$ and $\Delta$ to show how the decoupling of $\nu_{\mu, \tau}$ may occurs. It is worth noting that this form of $M_{L}$ is produced by the conservation of $L$ in the Standard Model which suggest that $M_{D}$ should be diagonal, thus giving $M_{L}$ as above. In this picture, the $L$ violation terms come from high energy, and all the texture in the mixing matrix is produced through $M_{N}$. Also, notice that the mass matrix (23) is really of a generical form. It may be produced not only by see-saw as we are assuming here but as well as through some other mechanism as radiative corrections.

Now, the main ingredient of the decoupling is based on the fact that the bulk neutrino really couples to a certain linear combination of the brane neutrinos, given by $M_{L} M_{N}^{-1}\left(\nu_{0 B}, \nu_{\mu}, \nu_{\tau}\right)_{L}^{\dagger}$, and decouples from the orthogonal combinations. Moreover, the first row and column of $M_{L} M_{N}^{-1} M_{L}^{\dagger}$ correspond to the same combination. Therefore by rotating 
the brane sector to diagonalize $M_{L} M_{N}^{-1} M_{L}^{\dagger}$, we may expect that the decoupling becomes explicit. Lets see how this argument works. First notice that if we make the rotation

$$
\left(\begin{array}{c}
\nu_{0 B}^{\prime} \\
\nu_{2} \\
\nu_{3}
\end{array}\right)_{L}=K\left(\begin{array}{c}
\nu_{0 B} \\
\nu_{\mu} \\
\nu_{\tau}
\end{array}\right)_{L}
$$

then the mass matrix (23) transforms into

$$
\left(\begin{array}{ccc}
K M_{L} M_{N}^{-1} M_{L}^{\dagger} K^{\dagger} & \sqrt{2} K M_{L} M_{N}^{-1} \mathbf{m} & 0 \\
\sqrt{2} \mathbf{m}^{\dagger} M_{N}^{-1} M_{L}^{\dagger} K^{\dagger} & 2 \mathbf{m}^{\dagger} M_{N}^{-1} \mathbf{m} & \partial_{5} \\
0 & \partial_{5} & 0
\end{array}\right)
$$

Let us choose $K$ such that $K M_{L} M_{N}^{-1} M_{L}^{\dagger} K^{\dagger}$ becomes diagonal. All the mixing with the bulk modes $\nu_{B L, R}^{\prime}$ are then given by the form of the column vector $K M_{L} M_{N}^{-1} \mathbf{m}$. It is here that we expect to see the decoupling. Let us take an explicit form for $M_{N}$ to make it clear:

$$
M_{N}=\left(\begin{array}{lll}
0 & a & 0 \\
a & 0 & b \\
0 & b & c
\end{array}\right)
$$

and to simplify matters let us assume that $m=10, m_{2}=50, m 3=100$ and $a=10^{7}$, $b=5 \times 10^{9}$ and $c=10^{8}$ all in $\mathrm{MeV}$. These values are selected merely for illustrative purpose. Other values may also achieve the desired decoupling. The correct entries in the matrix depend on the high scale sector of the model, which we leave unspecified for our purpose. Now we proceed with the numerical computation with above choice of numbers and get

$$
K=\left(\begin{array}{ccc}
0.9998 & 2 \times 10^{-4} & -2 \times 10^{-2} \\
-1.4 \cdot 10^{-2} & 0.7103 & -0.7038 \\
-1.4 \cdot 10^{-2} & -0.7039 & -0.7002
\end{array}\right)
$$

while the eigenvalues of $M_{L} M_{N}^{-1} M_{L}^{\dagger}$ are given by $(.25 \mathrm{MeV},-1.0028 \mathrm{eV}, 1.0008 \mathrm{eV})$. Thus we get a maximal mixing in the right sector. On the other hand, for the mixing with the bulk modes we get $K M_{L} M_{N}^{-1} \mathbf{m}=\left(.25,1.4 \cdot 10^{-8},-1.4 \cdot 10^{-8}\right) \mathrm{MeV}$. Thus, $\nu_{2,3}$ decouples while $\nu_{0 B L}^{\prime}$ mixes maximally to the bulk modes.

Next, as the entry $\mathbf{m}^{\dagger} M_{N}^{-1} \mathbf{m}$ in (23) also is the first element in the mixing $M_{L} M_{N}^{-1} \mathbf{m}$, after extracting the decoupling modes, the effective mass matrix reduces to the profile

$$
\left(\begin{array}{ccc}
\alpha & \sqrt{2} \alpha & 0 \\
\sqrt{2} \alpha & 2 \alpha & \partial_{5} \\
0 & \partial_{5} & 0
\end{array}\right) \equiv\left(\begin{array}{cccccc}
\alpha & \sqrt{2} \alpha & 0 & \sqrt{2} \alpha & 0 & \cdots \\
\sqrt{2} \alpha & 2 \alpha & \mu_{0} & 2 \alpha & 0 & \cdots \\
0 & \mu_{0} & 0 & 0 & 0 & \cdots \\
\sqrt{2} \alpha & 2 \alpha & 0 & 2 \alpha & 2 \mu_{0} & \cdots \\
0 & 0 & 0 & 2 \mu_{0} & 0 & \cdots \\
\vdots & \vdots & \vdots & \vdots & \vdots & \ddots
\end{array}\right)
$$

where $\alpha=\mathbf{m}^{\dagger} M_{N}^{-1} \mathbf{m}$. It is simple to check this fact in the case above since $\alpha \approx .25$ $\mathrm{MeV}$ too. It is worth noting that now all the fields involved are bulk modes. The rows 
and columns in the left hand side of (29) are labeled by $\left(\nu_{0 L}, \nu_{B L}^{\prime}, \nu_{B R}^{\prime}\right)$. On the right hand side we have explicitly written it in its true infinite mass matrix form labeled by $\left(\nu_{0 L}, \nu_{1 B L}, \nu_{1 B R}, \nu_{2 B L}, \nu_{2 B R}, \cdots\right)$.

We may worry about the high value of $\alpha$ since it could mean that $\nu_{0 B L}$ has a component with a large mass; however, as a result of the maximal mixing with the KK modes, that contribution will be suppressed as well as the other heavy elements of the tower are. The mechanism works exactly as in the one generational case of this class of models [21] where the $\nu_{L}$ which gets mixed with the bulk modes has a mass term in the $\mathrm{MeV}$ range [see Eq. (17)]. There, the heavy term is absorbed by the tower and as a result the masses of the KK modes are shifted down, while their contributions to $\nu_{L}$ becomes suppressed as $1 / n$. This suppresses the heavy field contribution by the number of levels below such a mass i.e. $\sim \alpha R$ $\mathrm{MeV}$, which is a large number. The lowest mode then becomes the main component of $\nu_{0 L}$ that appears in Eq. (21); We identify this mode as the sterile neutrino, $\nu_{4}$, used in solving the solar neutrino puzzle.

Let us demonstrate this by diagonalizing the mass matrix (29). We take advantage of this compact notation to write the characteristic equation as

$$
\left(\lambda^{2}-\partial_{5}^{2}\right)\left[\alpha-\lambda+\frac{2 \alpha \lambda^{2}}{\lambda^{2}-\partial_{5}^{2}}\right]=0
$$

which now reduce to $\pi \alpha R \cot (\pi \lambda R)=1$ and may be solved exactly to get the mass eigenvalues

$$
\lambda_{n}=\frac{\arctan (\pi \alpha R)}{\pi R}+\frac{n}{R} .
$$

where now $n \in \mathcal{Z}$, since also the right handed bulk fields are involved. Clearly, when $\alpha R \gg 1$ (as is the case here) the masses are just $\lambda_{n}=(2 n+1) \mu_{0} / 2$, which are now shifted down by $\frac{1}{2} \mu_{0}$. Also we note that the only masses of order of $\alpha$ correspond to $n \approx \pm \alpha R$ which were already in the tower; thus our massive term has been totally absorbed into the KK tower. Strictly speaking, there is an extra mode in the tower with infinite mass.

The corresponding eigenvectors are given by ( $\lambda$ are the eigenvalues)

$$
\nu(\lambda)=\frac{1}{\eta(\lambda)}\left[\nu_{0 B L}+\frac{\sqrt{2} \lambda^{2}}{\lambda^{2}-\partial_{5}^{2}} \nu_{B L}^{\prime}+\frac{\sqrt{2} \lambda \partial_{5}}{\lambda^{2}-\partial_{5}^{2}} \nu_{B R}^{\prime}\right] .
$$

with the normalization factor:

$$
\eta^{2}=\left(\frac{\lambda}{\alpha}\right)^{2}\left[1+(\pi \alpha R)^{2}\right] \approx(2 n+1)^{2}\left(\frac{\pi}{2}\right)^{2} .
$$

Notice that in our limit i.e. $\alpha R \gg 1$, the masses are degenerate, and occur in pairs with masses $\pm \lambda_{n}$. We may then recombine those states to get two Majorana neutrinos that form a Dirac neutrino of mass $\lambda_{n}$ of the form

$$
\tilde{\nu}_{n L}=\frac{\sqrt{2}}{\eta\left(\lambda_{n}\right)}\left[\nu_{0 B L}+\frac{\sqrt{2} \lambda_{n}^{2}}{\lambda_{n}^{2}-\partial_{5}^{2}} \nu_{B L}^{\prime}\right] ; \quad \tilde{\nu}_{n R}=\frac{1}{\eta\left(\lambda_{n}\right)} \frac{2 \lambda_{n} \partial_{5}}{\lambda_{n}^{2}-\partial_{5}^{2}} \nu_{B R}^{\prime}
$$


Now, we may rotate the system backwards to write down $\nu_{0 B L}$ in terms of the left handed mass eigenstates to get

$$
\nu_{0 B L}=\sum_{n=0}^{\infty} \frac{\sqrt{2}}{\eta\left(\lambda_{n}\right)} \tilde{\nu}_{n L} .
$$

Thus, as $\eta$ grows quadratically in $\lambda_{n}$, the main element of $\nu_{0 B L}$ is the lowest mode $\tilde{\nu}_{0 L}$ that we then identify as the sterile neutrino. Moreover, from (33) we see that the suppression over the other modes goes as $1 /(2 n+1)$, then the heavier modes get highly suppressed, since $n$ is also the number of eigenlevels below.

Finally, we may put all the ingredients together and calculate the effective mixing pattern at low energies. We have proceeded using a numerical analysis from the beginning to estimate masses and mixings directly from the mass matrix in (19), without the approximations we made in the former analysis to get more accurate values. The input values for our parameters are the same as before with $m_{1}=1 \mathrm{MeV}$ and we have taken $\mu_{0}=5 \times 10^{-3} \mathrm{eV}$ to fit properly $\Delta m_{\text {sol }}^{2}$, we then get

$$
\left(\begin{array}{l}
\nu_{e} \\
\nu_{\mu}
\end{array}\right)=\left(\begin{array}{cccc}
0.9948 & 0.0212 & -0.0072 & 0.0632 \\
0.0199 & -0.7037 & 0.7102 & 0.0013
\end{array}\right)\left(\begin{array}{l}
\nu_{1} \text { (massless) } \\
\nu_{2}(-1.0014 \mathrm{eV}) \\
\nu_{3}(1.003 \mathrm{eV}) \\
\nu_{4}\left(2.5151 \cdot 10^{-3} \mathrm{eV}\right)
\end{array}\right)
$$

where the masses are indicated between brackets. From these results we get

$$
\begin{aligned}
& \Delta m_{\text {sol }}^{2} \sim 6.326 \times 10^{-6} \mathrm{eV}^{2}, \quad \sin ^{2} 2 \theta_{\text {sol }} \sim 1.58 \times 10^{-2} \\
& \Delta m_{\text {atm }}^{2} \sim 3.23 \times 10^{-3} \mathrm{eV}^{2}, \quad \sin ^{2} 2 \theta_{\text {atm }} \sim .9991 \\
& \Delta m_{e \mu}^{2} \sim 1.0028 \mathrm{eV}^{2}, \quad \sin ^{2} 2 \theta_{e \mu} \sim 1.18 \times 10^{-3}
\end{aligned}
$$

These values are to be compared with the experimentally preferred values (11), (2) and (3). It clearly shows that the choice of the bulk neutrino as the sterile neutrino works quite well to explain observations. Although for the sake of explicit demonstration we have made a specific choice of parameters, there is a range of parameters in the mass matrix that will do equally well.

\section{CONCLUSIONS}

In conclusion, our analysis seems to demonstrate that one can use the bulk neutrino as a sterile neutrino needed to understand all neutrino oscillation observations. Despite the fact that it has an infinite set of KK excitations, only the lowest mode seems to play an important role and indeed gets identified as the sterile neutrino. It is worth emphasizing again the conceptual advantage of this approach which is that the ultralightness of the sterile neutrino has a geometric origin rather than from extra symmetries, as in the four dimensional models. What is also gratifying is that the apparent connection between submillimeter gravity

experiments and the neutrino puzzles seems to remain in the realistic implementations of this idea.

Acknowledgements. The work of RNM is supported by a grant from the National Science Foundation under grant number PHY-9802551. The work of APL is supported in part by CONACyT (México). 


\section{REFERENCES}

[1] Y. Fukuda et al., Phys. Rev. Lett. 81, (1998) 1562; idem. 81 (1998) 1158; Phys. Lett. B436 (1998) 33.

[2] K.S. Hirata et al., Phys. Lett. B280 (1992) 146; R. Becker-Szendy et al., Phys. Rev. D 46 (1992) 3720; W. W. M. Allison et al., Phys. Lett. B 391 (1997) 491; Y. Fukuda et al, Phys. Lett. B 335 (1994) 237.

[3] B. T. Cleveland et al. Nucl. Phys. B (Proc. Suppl.) 38 (1995) 47; K.S. Hirata et al., Phys. Rev. 44 (1991) 2241; GALLEX Collaboration, Phys. Lett. B388 (1996) 384; J. N. Abdurashitov et al., Phys. Rev. Lett. 77 (1996) 4708.

[4] Super-Kamiokande collaboration, Phys. Rev. Lett. 81 (1998)1562.

[5] C. Athanassopoulos et al. Phys. Rev. Lett. 75 (1995) 2650; C. Athanassopoulos et al. Phys. Rev. C 58 (1998) 2489.

[6] J. Bahcall, P. Krastev and Smirnov, Phys. Rev. D 58 (1998) 096016; G.L. Fogli, E. Lisi, D. Montanino, Astropart. Phys. 9 (1998) 119.

[7] L. Wolfestein, Phys. Rev. D 17 (1978) 2369; S.P. Mikheyev, A. Smirnov, Yad. Fiz. 42 (1985) 1441; Nuovo Cimento 9C (1986) 17.

[8] M. C. Gonzalez-Garcia et al., Phys.Rev. D58 (1998) 033004

[9] B. Armbruster et al., Phys. Rev. C 57 (1998) 3414.

[10] B. Achkar et al., Nuc. Phys. B434 (1995) 503.

[11] L. Borodousky et al., Phys. Rev. Lett. 68 (1992) 274.

[12] D.O. Caldwell and R.N. Mohapatra, Phys. Rev. D 48 (1993) 3259; J. Peltoniemi and J.W.F. Valle, Nucl. Phys. B406 (1993) 409.

[13] S. Bilenky, C. Giunti and W. Grimus, Eur. Phys. J. C 1 (1998) 247; Pramana 51 (1998) 51; S. Goswami, Phys. Rev. D 55 (1997) 2931; N. Okada and O. Yasuda, Int.J.Mod.Phys. A12 (1997) 36691.

[14] R. Foot and R. Volkas, Phys. Rev. D 52, 6595 (1995).

[15] Q. Liu and A. Smirnov, Nucl. Phys. B524 (1998) 505.

[16] For alternative models for the sterile neutrino see for instance: Z. Berezhiani and R. N. Mohapatra, Phys. Rev. D 52 (1995) 6607. K. Benakli and A. Smirnov, Phys. Rev. Lett. 79 (1997) 4314; P. Langacker, Phys. Rev. D58 (1998) 093017; D. Suematsu, Phys. Lett. B392 (1997) 413; E. J. Chun, A. Joshipura and A. Smirnov, Phys. Rev. D 54 (1996) 4654; E. Ma and P. Roy, Phys. Rev. D 52 (1995) 4342; M. Bando and K. Yoshioka, Prog. Theor. Phys. 100 (1998) 1239; N. Arkani-Hamed and Y. Grossman, Phys. Lett. B459 (1999) 179; E. Ma, Mod. Phys. Lett. A 11 (1996) 1893; A. Pérez-Lorenzana and W. A. Ponce, hep-ph/9812402; Z. Chacko and R. N. Mohapatra, hep-ph/9905388.

[17] N. Arkani-Hamed, S. Dimopoulos and G. Dvali, Phys. Lett. B429 (1998) 263; Phys. Rev. D 59 (1999) 086004; I. Antoniadis, S. Dimopoulos, G. Dvali, Nucl. Phys. B516 (1998) 70; N. Arkani-Hamed, S. Dimopoulos and J. March-Russell, hep-th/9809124.

[18] K.R. Dienes, E. Dudas and T. Gherghetta, hep-ph/9811428; N. Arkani-Hamed, S. Dimopoulos, G. Dvali and J. March-Russell, hep-ph/9811448.

[19] G. Dvali and A.Yu. Smirnov, hep-ph/9904211.

[20] A. Faraggi and M. Pospelov, Phys. Lett. B458 (1999) 237; A. Das and O. C. W. Kong, hep-ph/9907272; G. C. McLaughlin, J. N. Ng, hep-ph/9909558.

[21] R. N. Mohapatra, S. Nandi and A. Pérez-Lorenzana, hep-ph/9907520; Phys. Lett B (to appear). 
[22] M. Gell-Mann, P. Ramond and R. Slansky, in Supergravity, eds. P. van Niewenhuizen and D.Z. Freedman (North Holland 1979); T. Yanagida, in Proceedings of Workshop on Unified Theory and Baryon number in the Universe, eds. O. Sawada and A. Sugamoto (KEK 1979); R. N. Mohapatra and G. Senjanović, Phys. Rev. Lett. 44, 912 (1980).

[23] R. N. Mohapatra and A. Pérez-Lorenzana, hep-ph/9909389; Phys. Lett. B (to appear).

[24] For a recent review, see R. N. Mohapatra, hep-ph/9910365. 